\title{
ON THE TOPOLOGY OF THE SPACE OF RICCI-POSITIVE METRICS
}

\author{
BORIS BOTVINNIK, JOHANNES EBERT, AND DAVID J. WRAITH
}

\begin{abstract}
We show that the space $\mathcal{R}^{\mathrm{pRc}}\left(W_{g}^{2 n}\right)$ of metrics with positive Ricci curvature on the manifold $W_{g}^{2 n}:=\sharp^{g}\left(S^{n} \times S^{n}\right)$ has nontrivial rational homology if $n \not \equiv 3(\bmod 4)$ and $g$ are both sufficiently large. The same argument applies to $\mathcal{R}^{\mathrm{pRc}}\left(W_{g}^{2 n} \sharp N\right)$ provided that $N$ is spin and $W_{g}^{2 n} \sharp N$ admits a Ricci positive metric.
\end{abstract}

\section{INTRODUCTION}

Let $M$ be a smooth closed manifold of dimension $d$. We denote by $\mathcal{R}(M)$ the space of all Riemannian metrics on $M$ with the $C^{\infty}$-topology, and we let $\mathcal{R}^{\mathrm{pRc}}(M) \subset \mathcal{R}^{\mathrm{psc}}(M) \subset \mathcal{R}(M)$ be the subspaces of metrics with positive Ricci curvature and with positive scalar curvature respectively (abbreviated later as "pRc metrics" and "psc metrics").

In recent years, there has been a considerable effort to study the topology of the space of all positive scalar curvature metrics on manifolds, see [13, 2, 7, 6, 22]. The results achieved so far show that roughly the space $\mathcal{R}^{\mathrm{psc}}(M)$ at least is as complicated as real $K$-theory, provided one focuses on spin manifolds in dimensions at least five.

The aim of this paper is to study the space of Ricci positive metrics on certain manifolds. Very little is known about the topology of this space. It was shown in [27] that the space of Ricci positive metrics on all homotopy spheres which bound parallelisable manifolds in dimensions $4 n-1(n \geq 2)$ has infinitely many path-components. More recently, it was shown in [7] (discussed below) that for spin manifolds in dimension at least six, there are order two elements in certain higher homotopy groups of this space of metrics. We will supplement these results by showing that the space of Ricci positive metrics for certain manifolds can display non-trivial higher rational cohomology.

If $M$ is a spin manifold, there is a secondary index map inddiff $g: \mathcal{R}^{\mathrm{psc}}(M) \rightarrow \Omega^{\infty+d+1} \mathrm{KO}$, depending on the choice of a basepoint $g \in \mathcal{R}^{\text {psc }}(M)$ and mapping into a suitable space of the real $K$-theory spectrum. The map is constructed using the spin Dirac operator and the SchrödingerLichnerowicz formula. We refer to the introduction of [2] for an informal discussion and to [2, Section 3.3] for precise definitions. On homotopy groups, the map inddiff $g$ induces a homomorphism

$$
\left(\operatorname{inddiff}_{g}\right)_{*}: \pi_{m}\left(\mathcal{R}^{\mathrm{psc}}(M), g\right) \rightarrow K O_{d+m+1}:=K O_{d+m+1}(*),
$$

and it has been proven [2] that (1) is surjective after rationalization for each spin manifold of dimension $d \geq 6$ that admits a psc metric. More recently, Perlmutter [21, 22] showed how to cover the case $d=5$. The proof of these results relies heavily on a remarkable property of the space $\mathcal{R}^{\mathrm{psc}}(M)$ : a strengthening of the classical Gromov-Lawson surgery theorem [11] (see [5] and also

2000 Mathematics Subject Classification. 53C27, 57R65, 58J05, 58J50. 
[25]) shows that the homotopy type of $\mathcal{R}^{\mathrm{psc}}(M)$ only depends on the spin cobordism class of $M$ (for simply connected $M$ of dimension $d \geq 5$ ).

Positivity of the Ricci curvature is a much more restrictive condition than positivity of scalar curvature, and it is well-known that no variant of the Gromov-Lawson surgery theorem can hold for positive Ricci curvature, as any such variant would imply that $S^{1} \times S^{d-1}$ has positive Ricci curvature for large enough $d$, contradicting Myers' theorem [23, Theorem 25 on p. 171]. In particular, the general approach of the paper [2] showing the nontriviality of (1) does not work when the space $\mathcal{R}^{\mathrm{psc}}(M)$ is replaced by $\mathcal{R}^{\mathrm{pRc}}(M)$.

Another, older, approach to construct elements in $\mathcal{R}^{\text {psc }}(M)$ which was pioneered by Hitchin [15] has the advantage that it does not require elaborate constructions of metrics of positive scalar curvature, and clearly carries over to positive Ricci curvature. One uses the action of the diffeomorphism group $\operatorname{Diff}(M)$ or better the spin diffeomorphism group ${ }^{1} \operatorname{Diff}^{\text {spin }}(M)$ on $\mathcal{R}^{\mathrm{psc}}(M)$.

For a fixed $g_{0} \in \mathcal{R}^{\mathrm{psc}}(M)$, there is the orbit map ev $\operatorname{gv}_{0}: \operatorname{Diff}(M)^{\mathrm{spin}} \stackrel{f \mapsto f^{*} g_{0}}{\longrightarrow} \mathcal{R}^{\mathrm{psc}}(M)$, and we obtain the composition

$$
\pi_{m}\left(\operatorname{Diff}(M)^{\mathrm{spin}}\right) \stackrel{\left(\mathrm{ev}_{g_{0}}\right)_{*}}{\longrightarrow} \pi_{m}\left(\mathcal{R}^{\mathrm{psc}}(M)\right) \stackrel{\left(\operatorname{inddiff}_{g_{0}}\right)_{*}}{\longrightarrow} K O_{d+m+1} .
$$

The homomorphism (2) does not depend on the choice of $g_{0}$ (i.e., the two dependencies on $g_{0}$ cancel out) and can be computed by topological means, using the family index theorem. Clearly, the homomorphism (2) factors through $\pi_{m}\left(\mathcal{R}^{\mathrm{pRc}}(M)\right)$ as well, provided the fixed metric $g_{0}$ has positive Ricci curvature. Crowley-Schick-Steimle [7, Corollary 1.9] proved that if $d \geq 6$, there are elements in $\pi_{8 r+i-d}\left(\operatorname{Diff}^{\operatorname{spin}}(M)\right), i=0,1$, which under the homomorphism (2) hit the nontrivial element of $K \mathrm{O}_{8 r+i+1}=\mathbb{Z} / 2$, generalizing previous work by Hitchin [15] and Crowley-Schick [6].

If $m=4 k-d-1$, the target of the homomorphism (2) is $K O_{d+m+1}=\mathbb{Z}$. Then the homomorphism (2) can be computed by means of characteristic classes using the following recipe. Let $f: S^{4 k-d-1} \rightarrow \operatorname{Diff}^{\operatorname{spin}}(M)$ be a map representing an element $[f] \in \pi_{4 k-d-1}\left(\operatorname{Diff}^{\text {spin }}(M)\right)$. Since $\pi_{4 k-d-1}\left(\operatorname{Diff}^{\text {spin }}(M)\right) \cong \pi_{4 k-d}\left(B \operatorname{Diff}^{\text {spin }}(M)\right)$, we obtain a corresponding element in $\pi_{4 k-d}\left(B \operatorname{Diff}^{\text {spin }}(M)\right)$ represented by a map $\bar{f}: S^{4 k-d} \rightarrow B \operatorname{Diff}^{\text {spin }}(M)$, with $\bar{f}$ uniquely determined by $f$ up to homotopy. Using $\bar{f}$ to pull back the universal spin- $M$-bundl ${ }^{2}$, we form the spin- $M$-bundle $\pi: E_{f} \rightarrow S^{4 k-d}$ with vertical tangent bundle $T_{v} E_{f}$. Let $\hat{A}_{k}\left(T_{v} E_{f}\right) \in H^{4 k}\left(E_{f} ; \mathbb{Q}\right)$ be the degree $4 k$ component of the $\hat{A}$-class of $T_{v} E_{f}$. From this we can compute the tautological characteristic class

$$
\kappa_{\hat{A}_{k}}\left(E_{f}\right):=\pi_{!}\left(\hat{A}_{k}\left(T_{v} E_{f}\right)\right) \in H^{4 k-d}\left(S^{4 k-d} ; \mathbb{Q}\right)
$$

${ }^{1}$ Let us briefly recall the definition. Fix a spin structure on $s$ on $M$ and let $\widetilde{\operatorname{Diff}^{\text {spin }}}(M) \subset \operatorname{Diff}(M)$ be the (finite index open) subgroup of diffeomorphisms which fix $s$ up to isomorphism. Then Diff ${ }^{\text {spin }}(M)$ consists of all $(f, \hat{f})$, $f \in \widehat{\operatorname{Diff}^{\text {spin }}}(M)$ and $\hat{f}$ is an isomorphism $f^{*} s \cong s$ of spin structures. The group $\operatorname{Diff}{ }^{\text {spin }}(M)$ is a central extension of $\widetilde{\operatorname{Diff}^{\text {spin }}}(M)$ by $\mathbb{Z} / 2$.

${ }^{2}$ A spin- $M$-bundle is a fibre bundle with fibre $M$ and structure group Diff ${ }^{\text {spin }}(M)$. Equivalently, it is a smooth fibre bundle with fibre $M$, together with a spin structure on its vertical tangent bundle. 
and integrate over $S^{4 k-d}$ to obtain the $\hat{A}$-genus $\hat{A}(f) \in \mathbb{Q}$ of the homotopy class of $f$. The AtiyahSinger family index theorem implies that $\hat{A}(f)$ is an integer and that

$$
\left(\text { inddiff }_{g}\right)_{*}\left(\mathrm{ev}_{g}\right)_{*}([f])=a_{k} \hat{A}(f) \in K O_{4 k} \cong \mathbb{Z}
$$

where $a_{k}=1$ if $k$ is even and $a_{k}=\frac{1}{2}$ if $k$ is odd. We conclude: if $M$ admits a Ricci positive metric $g_{0}$ and if there is $f \in \pi_{d+1-4 k}\left(\operatorname{Diff}^{\mathrm{spin}}(M)\right)$ with $\hat{A}(f) \neq 0$, then $\pi_{d+1-4 k}\left(\mathcal{R}^{\mathrm{pRc}}(M)\right) \otimes \mathbb{Q} \neq 0$ (and (inddiff $\left.g_{0}\right)_{*} \neq 0$ in that degree).

However, it seems to be a very difficult problem to construct such elements in the homotopy groups of Diff ${ }^{\operatorname{spin}}(M)$ when the relevant $K O$-group is $\mathbb{Z}$. (It is known that such manifolds $M$ exist, by [13, Theorem 1.3], but the proof in that paper does not provide a concrete construction of $M$, let alone an $M$ which has a Ricci positive metric.)

In the present paper, we study the manifolds $W_{g}^{2 n}:=\left(S^{n} \times S^{n}\right)^{\# g}$, the connected sum of $g$ copies of $S^{n} \times S^{n}$. It has been proven by Sha and Yang [24, Theorem 1] that these admit a metric of positive Ricci curvature, if $n \geq 2$. It is known that for each $f \in \pi_{*}\left(\operatorname{Diff}^{\operatorname{spin}}\left(W_{g}^{2 n}\right)\right)$, the $\hat{A}$-genus is trivial, i.e. $\hat{A}(f)=0$. This follows from [13, Proposition 1.9] since the Pontrjagin classes of $W_{g}^{2 n}$ are all trivial. Hence with the method just described, one cannot detect nontrivial elements in $\pi_{*}\left(\mathcal{R}^{\mathrm{pRc}}\left(W_{g}^{2 n}\right)\right) \otimes \mathbb{Q}$. What we do in this paper is to prove that the rational cohomology $H^{j}\left(\mathcal{R}^{\mathrm{pRc}}\left(W_{g}^{2 n}\right) ; \mathbb{Q}\right)$ is nontrivial, for some values of $g, n$ and $j$.

Definition 1.1. Let $M$ be a closed manifold of dimension $2 n$. The genus $g(M)$ of $M$ is the largest number $g$ so that $M$ can be written as a connected sum $N \sharp W_{g}^{2 n}$ for some smooth manifold $N$.

Let us state our main result.

Theorem A. Let $M^{2 n}$ be a closed spin manifold of genus $g$ and assume that $n \not \equiv 3(\bmod 4)$. Assume that $M$ admits a metric of positive Ricci curvature. Then

(1) If $n=4 q \geq 14$ and $g \geq 21$, then $H^{j}\left(\mathcal{R}^{\mathrm{pRc}}(M) ; \mathbb{Q}\right) \neq 0$ for some $1 \leq j \leq 7$.

(2) If $n=4 q+1 \geq 11$ and $g \geq 17$, then $H^{j}\left(\mathcal{R}^{\mathrm{pRc}}(M) ; \mathbb{Q}\right) \neq 0$ for some $1 \leq j \leq 5$.

(3) If $n=4 q+2 \geq 8$ and $g \geq 13$, then $H^{j}\left(\mathcal{R}^{\mathrm{pRc}}(M)\right.$; $\left.\mathbb{Q}\right) \neq 0$ for some $1 \leq j \leq 3$.

Even though we use the index theory of the Dirac operator, our proof does not yield the nontriviality of (inddiff $\left.g_{0}\right)_{*}$ on homotopy or homology groups.

Using the known constructions of Ricci positive metrics on manifolds of large genus, we obtain the following list of examples to which Theorem A applies. Note that all examples admit spin structures and are shown to have Ricci positive metrics in the sources quoted.

Corollary 1.2. The conclusion of Theorem $A$ holds for the manifold $M \sharp W_{g}^{2 n}$ (for $g$ and $n$ as in Theorem A) if $M$ is any manifold from the following list.

(1) $M=S^{2 n}[24$, Theorem 1]; 
(2) $M$ is a connected sum with summands of the form $\mathbb{C} P^{d / 2} \times S^{\mathbf{d}_{I}}, \mathbb{H} P^{d / 4} \times S^{\mathbf{d}_{I}}, \mathbb{O} P^{2} \times S^{\mathbf{d}_{I}}$, $S^{d} \times S^{\mathbf{d}_{I}}$, where $S^{\mathbf{d}_{I}}$ denotes the product of spheres $S^{d_{1}} \times \cdots \times S^{d_{s}}$ with $d_{i} \geq 3$, and $d \geq 2$. The quantities $d$ and $\mathbf{d}_{I}$ are allowed to vary from summand to summand, $d$ must be a multiple of four in the second case, and an odd multiple of two in the first case (to ensure that $\mathbb{C} P^{d / 2}$ is spin). [3, Theorem A], but see also [26, Theorem B] and [4, Corollary 4.10].

(3) $M=N \sharp\left(S^{q_{1}} \times S^{r_{1}}\right) \sharp \cdots \sharp\left(S^{q_{m}} \times S^{r_{m}}\right)$, where $q_{i}+r_{i}=2 n, q_{i}, r_{i} \geq 3$ for each $i$, and $N$ is an $S^{a}$-bundle over a Ricci positive base space $X^{b}$ with compact Lie structure group, $a+b=2 n$, and $a>b \geq 3$ [26, Theorem B];

(4) $M=N \sharp E_{1} \sharp \cdots \sharp E_{m}$, with $N$ as in (3) but satisfying the tighter dimensional restriction $a \geq b+5$, and where $E_{1}, \cdots, E_{m}$ are linear sphere bundles with base $S^{c_{i}}$, fibre $S^{2 n-c_{i}}$, and $a-1 \geq c_{i} \geq b+4[26$, Theorem $\mathrm{C}]$;

1.1. Acknowledgments. We would like to thank Achim Krause for pointing out a counterexample to an erronous argument in the first draft of this paper, and Manuel Krannich for spotting a mistake in the first preprint version. Furthermore, we thank the referee for suggesting a simpler exposition at various places in this paper.

\section{ProOFS}

2.1. The detection theorem. The proof of Theorem A is based on the following general detection principle.

Theorem 2.1. Let $M$ be a d-dimensional closed smooth spin manifold. Assume that

(1) $M$ has a metric of positive Ricci curvature,

and that there is a smooth $M$-bundle $\pi: E \rightarrow B$ with a spin structure on its vertical tangent bundle $T_{v}$ E, such that

(2) the base space $B$ is 0-connected and has finite fundamental group, and

(3) for some $k>d / 4$, the tautological class $\kappa_{\hat{A}_{k}}(E) \in H^{4 k-d}(B ; \mathbb{Q})$ associated to the $k$-th component of the $\hat{A}$-class is nonzero.

Then the cohomology group $H^{j}\left(\mathcal{R}^{\mathrm{pRc}}(M) ; \mathbb{Q}\right)$ is nontrivial for some $1 \leq j \leq 4 k-d-1$.

Remark 2.2. The proof applies verbatim to any other curvature condition which implies positivity of scalar curvature, e.g. positive sectional curvature. We show below that a manifold as in Theorem A satisfies the hypotheses (2) and (3). On the other hand, manifolds of large genus do not admit metrics of positive sectional curvature, by Gromov's Betti number estimate [12, Theorem 0.2.A]. More generally, we are not aware of any example of a manifold $M$ which carries a metric of positive sectional curvature and satisfies the hypotheses (2) and (3) of Theorem 2.1 . 
Proof of Theorem 2.1. Consider the universal cover $p: \tilde{B} \rightarrow B$. Since $\pi_{1}(B)$ is a finite group, it follows from a transfer argument [14, Proposition 3G.1] that the induced homomorphism

$$
p^{*}: H^{*}(B ; \mathbb{Q}) \rightarrow H^{*}(\tilde{B} ; \mathbb{Q})
$$

is injective. In particular, this means that the pull-back spin- $M$-bundle $p^{*} E \rightarrow \tilde{B}$ satisfies the hypotheses of the theorem, and we can assume without loss of generality that the base $B$ is simplyconnected.

Associated with the fibre bundle $\pi: E \rightarrow B$, there is a fibration

$$
T^{\mathrm{pRc}}(\pi) \stackrel{\Pi}{\rightarrow} B
$$

whose fibre over $b \in B$ is the space $\mathcal{R}^{\mathrm{pRc}}\left(\pi^{-1}(b)\right)$. The construction of that fibration is as follows: there is a $\operatorname{Diff}(M)$-principal bundle $Q \rightarrow B$ such that $Q \times_{\operatorname{Diff}(M)} M \cong E$ (there is no need to take the spin condition into account here). We define

$$
T^{\mathrm{pRc}}(\pi):=Q \times \operatorname{Diff}(M) \mathcal{R}^{\mathrm{pRc}}(M) .
$$

The pullback $M$-bundle $\Pi^{*} E \rightarrow T^{\mathrm{pRc}}(\pi)$ admits a spin structure since the original fibre bundle $\pi: E \rightarrow B$ does, and in addition it has a fibrewise Riemannian metric of positive Ricci curvature, and hence of positive scalar curvature.

Now let $\operatorname{ind}_{E} \in K O^{-d}(B)$ be the index class of the fibrewise Dirac operator on $E$. The Schrödinger-Lichnerowicz formula implies that $\Pi^{*} \operatorname{ind}_{E}=0 \in K O^{-d}\left(T^{\mathrm{pRc}}(\pi)\right)$. Under the composition

$$
K O^{-d}(B) \stackrel{c}{\rightarrow} K^{-d}(B) \stackrel{\mathrm{ch}_{2 k}}{\rightarrow} H^{4 k-d}(B ; \mathbb{Q})
$$

of the complexification map with the $2 k$ th component of the Chern character, the class ind $E$ is mapped to the tautological class $\kappa_{\hat{A}_{k}}(E)$ (this is the cohomological version of the Atiyah-Singer family index theorem for the spin Dirac operator). Therefore, we obtain that

$$
\Pi^{*} \kappa_{\hat{A}_{k}}(E)=\operatorname{ch}_{2 k}\left(c\left(\Pi^{*} \operatorname{ind}_{E}\right)\right)=0 \in H^{4 k-d}\left(T^{\mathrm{pRc}}(\pi) ; \mathbb{Q}\right) .
$$

In other words: the nontrivial element $\kappa_{\hat{A}_{k}}(E) \in H^{4 k-d}(B ; \mathbb{Q})$ lies in the kernel of $\Pi^{*}$. Now we consider the Serre spectral sequence of the fibration (3). The homomorphism $\Pi^{*}: H^{*}(B ; \mathbb{Q}) \rightarrow$ $H^{*}\left(T^{\mathrm{pRc}}(\pi)\right)$ agrees with the composition

$$
H^{4 k-d}(B ; \mathbb{Q}) \stackrel{\iota}{\longrightarrow} H^{4 k-d}\left(B ; H^{0}\left(\mathcal{R}^{\mathrm{pRc}}(M) ; \mathbb{Q}\right)\right)=E_{2}^{4 k-d, 0} \longrightarrow E_{4 k-d+1}^{4 k-d, 0} \longrightarrow H^{4 k-d}\left(T^{\mathrm{pRc}}(\pi)\right) .
$$

The first homomorphism $\iota$ is injective: it is induced from the injective (it is here that the hypothesis $\mathcal{R}^{\mathrm{pRc}}(M) \neq \emptyset$ is used) map $\mathbb{Q} \rightarrow H^{0}\left(\mathcal{R}^{\mathrm{pRc}}(M) ; \mathbb{Q}\right)$ of coefficient systems over $B$. Since the base $B$ is simply connected, an injective map of rational coefficient systems is split-injective and therefore induces an injective map in cohomology.

Then it follows that for some $2 \leq r \leq 4 k-d$, the differential $d_{r}: E_{r}^{4 k-d-r, r-1} \rightarrow E_{r}^{4 k-d, 0}$ is nonzero. Hence $E_{r}^{4 k-d-r, r-1} \neq 0$, which forces $H^{4 k-d-r}\left(B ; H^{r-1}\left(\mathcal{R}^{\mathrm{pRc}}(M) ; \mathbb{Q}\right)\right)=E_{2}^{4 k-d-r, r-1} \neq 0$. In particular $H^{r-1}\left(\mathcal{R}^{\mathrm{pRc}}(M) ; \mathbb{Q}\right) \neq 0$, as desired. 
2.2. The Torelli group of $W_{g}^{2 n}$ and its cohomology. We now give the proof of Theorem A. It relies on computations by the second-named author and Randal-Williams [8] which by themselves rely on many deep ingredients from high-dimensional manifold theory, such as [10] and [9], among other things.

Definition 2.3. Let $D \subset W_{g}^{2 n}$ be a fixed embedded closed $2 n$-disk, and let $\operatorname{Diff}\left(W_{g}^{2 n}, D\right)$ denote the group of diffeomorphisms of $W_{g}^{2 n}$ which restrict to the identity on some open neighborhood of the disk $D$. The Torelli group $\mathfrak{T}_{\mathfrak{c}}^{2 n}$ is the group of diffeomorphisms $\phi \in \operatorname{Diff}\left(W_{g}^{2 n}, D\right)$ which induce the identity in the middle homology group of $W_{k}^{2 n}$ :

$$
\mathfrak{T o r}_{g}^{2 n}:=\left\{\phi \mid \phi_{*}=\mathrm{id}: H_{n}\left(W_{g}^{2 n} ; \mathbb{Z}\right) \rightarrow H_{n}\left(W_{g}^{2 n} ; \mathbb{Z}\right)\right\} \subset \operatorname{Diff}\left(W_{g}^{2 n}, D\right) .
$$

Clearly the inclusion $\mathfrak{T o r}_{g}^{2 n} \subset \operatorname{Diff}\left(W_{g}^{2 n}, D\right)$ induces a map $\eta: B \mathfrak{T o r}{ }_{g}^{2 n} \rightarrow \operatorname{BDiff}\left(W_{g}^{2 n}, D\right)$ of the corresponding classifying spaces. There is a fibre sequence

$$
B \mathfrak{T o r}_{g}^{2 n} \stackrel{\eta}{\rightarrow} \operatorname{BDiff}\left(W_{g}^{2 n}, D\right) \rightarrow B \Gamma\left(W_{g}^{2 n}\right),
$$

where $\Gamma\left(W_{g}^{2 n}\right) \subset \mathrm{GL}\left(H_{n}\left(W_{g}^{2 n} ; \mathbb{Z}\right)\right)$ is a certain arithmetic group, see [8, Section 2.1].

Lemma 2.4. Let $n \geq 4$ and $n \not \equiv 3(\bmod 4)$. Then $\pi_{0}\left(\mathfrak{T o r}_{g}^{2 n}\right)$ is a finite group.

Proof. This is a corollary of a result by Kreck [17, Theorem 2]. Kreck studies the group of orientation-preserving diffeomorphisms $\operatorname{Diff}^{+}\left(W_{g}^{2 n}\right)$ (not just those preserving a disk) and the subgroup of diffeomorphisms acting as the identity on $H_{n}\left(W_{g}^{2 n}\right)$, which he denotes by $S \operatorname{Diff}\left(W_{g}^{2 n}\right)$. There is an analogous fibre sequence $B S \operatorname{Diff}\left(W_{g}^{2 n}\right) \rightarrow B \operatorname{Diff}^{+}\left(W_{g}^{2 n}\right) \rightarrow B \Gamma\left(W_{g}^{2 n}\right)$, and the comparison with the fibre sequence (5) yields the following diagram of groups

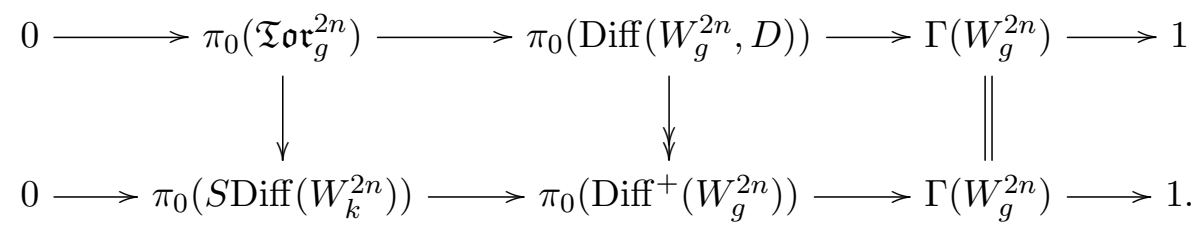

Kreck showed that the lower sequence is exact [17, Theorem 2], and the upper one is exact as well, see [8, Proposition 2.3]. It is part of the proof of [8, Proposition 2.3] that the middle vertical map is surjective. Using the fibre sequence

$$
\operatorname{Diff}\left(W_{g}^{2 n}, D\right) \rightarrow \operatorname{Diff}^{+}\left(W_{g}^{2 n}\right) \rightarrow \operatorname{Emb}^{+}\left(D, W_{k}^{2 n}\right),
$$

the weak homotopy equivalence ${ }^{3} \operatorname{Emb}^{+}\left(D, W_{g}^{2 n}\right) \simeq \operatorname{Fr}^{+}\left(T W_{g}^{2 n}\right)$ of the space of orientation-preserving embeddings of $D$ to the oriented frame bundle of $W_{g}^{2 n}$, and the fact that $\operatorname{Fr}^{+}\left(T W_{g}^{2 n}\right)$ is connected and has $\pi_{1}\left(\operatorname{Fr}^{+}\left(T W_{g}^{2 n}\right)\right) \cong \mathbb{Z} / 2$, we deduce that the kernel of the middle vertical map in (6) has order $\leq 2$, and hence

$$
\pi_{0}\left(\mathfrak{T o r}_{g}^{2 n}\right) \rightarrow \pi_{0}\left(S \operatorname{Diff}\left(W_{k}^{2 n}\right)\right)
$$

\footnotetext{
${ }^{3}$ This is a standard fact: the inclusion $\operatorname{Emb}^{+}\left(D, W_{g}^{2 n}\right) \rightarrow \operatorname{Imm}^{+}\left(D, W_{g}^{2 n}\right)$ into the space of orientation-preserving immersions is a weak equivalence by a scaling argument, and the map $\operatorname{Imm}^{+}\left(D, W_{g}^{2 n}\right) \rightarrow \operatorname{Fr}^{+}\left(W_{g}^{2 n}\right)$ that takes the derivative at the origin is a weak homotopy equivalence by the Smale-Hirsch immersion theorem.
} 
is surjective with finite kernel. Kreck [17, Theorem 2] shows moreover the existence of an exact sequence $0 \rightarrow \Theta_{2 n+1} / \Sigma \rightarrow \pi_{0}\left(S \operatorname{Diff}\left(W_{k}^{2 n}\right)\right) \rightarrow \operatorname{Hom}\left(H_{n}\left(W_{g}^{2 n} ; \mathbb{Z}\right) ; S \pi_{n}(S O(n))\right) \rightarrow 0$, where $\Theta_{2 n+1}$ is the group of homotopy spheres which is finite by [16] and $\Sigma$ is a certain subgroup, and $S \pi_{n}(S O(n)) \subset \pi_{n}(S O(n+1))$ is the image of the stabilization map $S O(n) \rightarrow S O(n+1)$. The values of $S \pi_{n}(S O(n))$ are tabularized in [17, p. 644], and from that table, one reads off that $\operatorname{Hom}\left(H_{n}\left(W_{g}^{2 n} ; \mathbb{Z}\right) ; S \pi_{n}(S O(n))\right)$ is finite unless $n \equiv 3(\bmod 4)$.

The hard part is now to show that the tautological classes $\kappa_{\hat{A}_{k}} \in H^{4 k-2 n}\left(B \mathfrak{T o r}_{g}^{2 n} ; \mathbb{Q}\right)$ are nontrivial. This was essentially done in [8], and we only recall the main points. Consider the graded algebra $\mathbb{Q}\left[p_{\left\lceil\frac{n+1}{4}\right\rceil}, \ldots, p_{n}, e\right] /\left(e^{2}-p_{n}\right)$, which is the rational cohomology algebra of the $n$ connected cover of $B O(2 n)$. Define a graded vector space by

$$
\left(\sigma^{-2 n} \mathbb{Q}\left[p_{\left\lceil\frac{n+1}{4}\right\rceil}, \ldots, p_{n}, e\right] /\left(e^{2}-p_{n}\right)\right)_{j}:= \begin{cases}\left(\mathbb{Q}\left[p_{\left\lceil\frac{n+1}{4}\right\rceil}, \ldots, p_{n}, e\right] /\left(e^{2}-p_{n}\right)\right)_{j+2 n} & j>0, \\ 0 & j \leq 0 .\end{cases}
$$

Assigning to $c \in \sigma^{-2 n} \mathbb{Q}\left[p_{\left\lceil\frac{n+1}{4}\right\rceil}, \ldots, p_{n}, e\right] /\left(e^{2}-p_{n}\right)$ the tautological class $\kappa_{c}$ of the universal $W_{g}^{2 n}$ bundle defines a map of graded vector spaces

$$
\sigma^{-2 n} \mathbb{Q}\left[p_{\left\lceil\frac{n+1}{4}\right\rceil}, \ldots, p_{n}, e\right] /\left(e^{2}-p_{n}\right) \rightarrow H^{*}\left(B \operatorname{Diff}\left(W_{g}^{2 n}\right) ; \mathbb{Q}\right),
$$

which extends to a map from the free graded commutative algebra on the source. The main results of [9] and [10] show that this map is an isomorphism in degrees $j \leq(g-5) / 2$.

Now let $L_{k} \in \mathbb{Q}\left[p_{\left\lceil\frac{n+1}{4}\right\rceil}, \ldots, p_{n}, e\right] /\left(e^{2}-p_{n}\right)$ be the $k$ th component of the Hirzebruch $L$-class. It is a consequence of the Atiyah-Singer family index theorem for the signature operator that $\kappa_{L_{k}} \in H^{4 k-2 n}\left(B \operatorname{Diff}\left(W_{g}^{2 n}\right) ; \mathbb{Q}\right)$ maps to zero in $H^{*}\left(B \mathfrak{T o r}{ }_{g}^{2 n} ; \mathbb{Q}\right)$, see [8, Theorem 2.9]. We remark that in loc.cit., the version $\mathcal{L}_{k}$ of the $L$-class introduced by Atiyah and Singer [1, p. 577] is used, which is more natural from the viewpoint of the index theorem. The distinction is not important for us, since the two classes are related by the formula $\mathcal{L}_{k}=\frac{1}{2^{2 k}} L_{k}$.

Theorem 2.5 (Ebert, Randal-Williams [8]). Let $C_{g}^{2 n}$ be the largest integer with

(1) $C_{g}^{2 n} \leq(g-5) / 2$,

(2) $C_{g}^{2 n} \leq n-3$,

(3) $\max \left\{2 C_{g}^{2 n}+7,3 C_{g}^{2 n}+4\right\} \leq 2 n$.

Then the map

$$
V:=\frac{\sigma^{-2 n} \mathbb{Q}\left[p_{\left\lceil\frac{n+1}{4}\right\rceil}, \ldots, p_{n}, e\right] /\left(e^{2}-p_{n}\right)}{\operatorname{span}\left\{L_{k} \mid k \in \mathbb{N}\right\}} \rightarrow H^{*}\left(B \mathfrak{T} \mathfrak{o r}_{g}^{2 n} ; \mathbb{Q}\right)
$$

is injective in degrees $* \leq C_{g}^{2 n}$.

Remark 2.6. Theorem 2.5 is not stated as such in [8]. It is shown in [8, §5] that a certain map $B \mathfrak{T o r}_{g}^{2 n} \rightarrow \Omega^{\infty} F$ to a certain infinite loop space induces an isomorphism $H^{*}\left(\Omega^{\infty} F ; \mathbb{Q}\right) \rightarrow$ $\left.H^{*}(B \mathfrak{T o r})_{g}^{2 n} ; \mathbb{Q}\right)^{\Gamma\left(W_{g}^{2 n}\right)}$ in degrees $* \leq C_{g}^{2 n}$. Hence a fortiori, $H^{*}\left(\Omega^{\infty} F ; \mathbb{Q}\right) \rightarrow H^{*}\left(B \mathfrak{T o r}{ }_{g}^{2 n} ; \mathbb{Q}\right)$ is 
injective. The rational cohomology of $\Omega^{\infty} F$ is the free graded-commutative $\mathbb{Q}$-algebra generated by the left-hand side of (7). This is discussed in [8, $\S 5, \S 2.4]$.

Remark 2.7. Recently, Kupers and Randal-Williams [18, Theorem A], [19, Theorem A] gave a complete computation of $H^{*}\left(B \mathfrak{T o r}_{g}^{2 n} ; \mathbb{Q}\right)$, valid for all $n \geq 3$ and large $g$. Even though it is far from obvious from the results stated in [18], their calculations imply that the map (7) is injective if $* \leq(g-5) / 2$ and $n \geq 3$. This allows the lower bounds on $n$ in Theorem $\mathrm{A}$ to be replaced by $n \geq 3$.

Lemma 2.8. If $k \geq 2\left\lceil\frac{n+1}{4}\right\rceil$, the image of $\hat{A}_{k}$ in the quotient space $V$ on the left hand side of (7) is nonzero.

Proof. This follows from a characteristic class computation that has been carried out in [13, Section 5]. If $k \geq 2\left\lceil\frac{n+1}{4}\right\rceil$, it can be written in the form $k=a+b$ with $\left\lceil\frac{n+1}{2}\right\rceil \leq a \leq b$. Consider the quotient $V^{\prime}$ of $V$ by the subspace generated by all monomials except $p_{k}$ and $p_{a} p_{b}$ (note that $\operatorname{dim}\left(V^{\prime}\right) \leq 2$ ). By [13, Proposition 5.3], the image of $\hat{A}_{k}$ in $V^{\prime}$ is nonzero and hence it must be nonzero in $V$.

Proof of Theorem $A$. Write $M=N \sharp W_{g}^{2 n}$. The group $\operatorname{Diff}\left(W_{g}^{2 n} ; D\right)=\operatorname{Diff}_{\partial}\left(W_{g}^{2 n} \backslash D\right)$ and hence its subgroup $\mathfrak{T o r}_{g}^{2 n}$ acts by diffeomorphisms on $M$ : extend diffeomorphisms by the identity over all of $M$. Consider the fibre bundles

$$
E_{0}:=E \mathfrak{T o r}{ }_{g}^{2 n} \times \mathfrak{T o r}_{g}^{2 n} W_{g}^{2 n} \rightarrow B \mathfrak{T o r}_{g}^{2 n}=: B
$$

and

$$
E:=E \mathfrak{T o r}_{g}^{2 n} \times_{\mathfrak{T o r}_{g}^{2 n}} M \rightarrow B
$$

with fibres $W_{g}^{2 n}$ and $M$, respectively. An obstruction theory argument (see e.g. [2, Lemma 3.29]) shows that these bundles admit spin structures (since the structure group fixes a disc in the fibre, which is connected). By Lemma 2.4, $B$ has finite fundamental group.

Depending on the value of $n$ modulo 4 , consider the smallest $k_{0} \in \mathbb{N}$ such that $k_{0} \geq 2\left\lceil\frac{n+1}{4}\right\rceil$; the class $\hat{A}_{k_{0}}$ is an element in the degree $q_{0}=4 k_{0}-2 n$ part of the space $V$. Then $q_{0}=8,6,4$ in the cases $n \equiv 0,1,2(\bmod 4)$. For $n$ and $g$ obeying the bounds stated in Theorem A, it follows from Theorem 2.5 and Lemma 2.8 that the tautological class $\kappa_{\hat{A}_{k_{0}}}\left(E_{0}\right) \in H^{q_{0}}(B ; \mathbb{Q})$ is nontrivial.

The bundle $E$ is fibrewise cobordant to the disjoint union of $E_{0}$ with the trivial bundle $B \times N$. The classical argument [20, Lemma 17.3] for the cobordism invariance of Pontrjagin numbers shows that

$$
\kappa_{\hat{A}_{k}}(E)=\kappa_{\hat{A}_{k}}\left(E_{0}\right)+\kappa_{\hat{A}_{k}}(B \times N)=\kappa_{\hat{A}_{k}}\left(E_{0}\right)
$$

( since $4 k-2 n>0$, the second summand is zero). Hence all hypotheses of Theorem 2.1 are satisfied, and the proof is complete. 


\section{REFERENCES}

[1] M. F. Atiyah and I. M. Singer. The index of elliptic operators. III. Ann. of Math. (2), 87:546-604, 1968.

[2] B. Botvinnik, J. Ebert, and O. Randal-Williams. Infinite loop spaces and positive scalar curvature. Invent. Math., 209(3):749-835, 2017.

[3] B. Burdick. Ricci-positive metrics on connected sums of products with arbitrarily many spheres. ArXiv e-prints, March 2019.

[4] B. Burdick. Ricci positive metrics on connected sums of projective spaces. Differential Geometry and its Applications, 62:212-233, 2019.

[5] V. Chernysh. On the homotopy type of the space $\mathcal{R}^{+}(M)$. arXiv:math/0405235, 2004.

[6] D. Crowley and T. Schick. The Gromoll filtration, KO-characteristic classes and metrics of positive scalar curvature. Geom. Topol., 17(3):1773-1789, 2013.

[7] Diarmuid Crowley, Thomas Schick, and Wolfgang Steimle. Harmonic spinors and metrics of positive curvature via the Gromoll filtration and Toda brackets. J. Topol., 11(4):1077-1099, 2018.

[8] J. Ebert and O. Randal-Williams. Torelli spaces of high-dimensional manifolds. J. Topol., 8(1):38-64, 2015.

[9] S. Galatius and O. Randal-Williams. Stable moduli spaces of high-dimensional manifolds. Acta Math., 212(2):257-377, 2014.

[10] S. Galatius and O. Randal-Williams. Homological stability for moduli spaces of high dimensional manifolds. I. J. Amer. Math. Soc., 31(1):215-264, 2018.

[11] M. Gromov and H. B. Lawson. The classification of simply connected manifolds of positive scalar curvature. Ann. of Math. (2), 111(3):423-434, 1980.

[12] Michael Gromov. Curvature, diameter and Betti numbers. Comment. Math. Helv., 56(2):179-195, 1981.

[13] B. Hanke, T. Schick, and W. Steimle. The space of metrics of positive scalar curvature. Publ. Math. Inst. Hautes Études Sci., 120:335-367, 2014.

[14] Allen Hatcher. Algebraic topology. Cambridge University Press, Cambridge, 2002.

[15] Nigel Hitchin. Harmonic spinors. Advances in Math., 14:1-55, 1974.

[16] Michel A. Kervaire and John W. Milnor. Groups of homotopy spheres. I. Ann. of Math. (2), 77:504-537, 1963.

[17] M. Kreck. Isotopy classes of diffeomorphisms of $(k-1)$-connected almost-parallelizable $2 k$-manifolds. In Algebraic topology, Aarhus 1978 (Proc. Sympos., Univ. Aarhus, Aarhus, 1978), volume 763 of Lecture Notes in Math., pages 643-663. Springer, Berlin, 1979.

[18] Alexander Kupers and Oscar Randal-Williams. On the cohomology of Torelli groups. arXiv e-prints, page arXiv:1901.01862, Jan 2019.

[19] Alexander Kupers and Oscar Randal-Williams. The cohomology of Torelli groups is algebraic. arXiv e-prints, page arXiv:1908.04724, Aug 2019.

[20] John W. Milnor and James D. Stasheff. Characteristic classes. Princeton University Press, Princeton, N. J., 1974. Annals of Mathematics Studies, No. 76.

[21] N. Perlmutter. Cobordism Categories and Parametrized Morse Theory. ArXiv e-prints, March 2017.

[22] N. Perlmutter. Parametrized Morse Theory and Positive Scalar Curvature. ArXiv e-prints, May 2017.

[23] Peter Petersen. Riemannian geometry, volume 171 of Graduate Texts in Mathematics. Springer, New York, second edition, 2006.

[24] Ji-Ping Sha and DaGang Yang. Positive Ricci curvature on the connected sums of $S^{n} \times S^{m}$. J. Differential Geom., 33(1):127-137, 1991.

[25] M. Walsh. Cobordism invariance of the homotopy type of the space of positive scalar curvature metrics. Proc. Amer. Math. Soc., 141(7):2475-2484, 2013.

[26] D. J. Wraith. New connected sums with positive Ricci curvature. Ann. Glob. Anal. Geom., 32:343-360, 2007.

[27] D. J. Wraith. On the moduli space of positive Ricci curvature metrics on homotopy spheres. Geom. Topol., 15(4):1983-2015, 2011. 
Department of Mathematics, University of Oregon, Eugene, OR, 97403, USA

E-mail address: botvinn@uoregon.edu

Mathematisches Institut der Westfälischen Wilhelms-Universität Münster, Einsteinstr. 62, DE48149 Münster, Germany

E-mail address: johannes.ebert@uni-muenster.de

Department of Mathematics and Statistics, National University of Ireland Maynooth, Maynooth, IRELAND

E-mail address: david.wraith@mu.ie 